OUR ASTRONOMICAL COLUMN.

The Nature of Sun-Spots.-A useful summary of our knowledge of the phenomena presented by sunspots is given by the Rev. A. L. Cortie in Science Progress for October. A spot is regarded as an uprush of metallic vapours, which become cooled by rapid expansion, so that the spot appears dark by contrast with the bright solar surface. The umbra is considered to rise above the level of the photosphere, while the penumbra is built up by dark radial streams flowing from the umbra and seeking a level slightly lower than that of the photosphere. In round spots the penumbra is a shallow, saucer-like cavity, the lowest portion being due to the falling-in of the photospheric clouds caused by the initial uprush. This falling-in and heaping together of the photospheric clouds to fill the partial void produced by the ejection of the umbral vapours would account for the bright border which is generally seen to separate the umbra and penumbra. At a high level above the spot are the hydrogen flocculi, the rotation of which gives rise to the appearance of the solar vortices. Friction of the gyrating gases and vapours is considered competent to generate electric currents, and the accompanying magnetic fields which produce the Zeeman effects in the spectra of spots. Father Cortie considers it doubtful whether the umbra and penumbra of the spots themselves share this gyratory motion.

Solar Prominences in I9I5.-An account of the observations of solar prominences made at Catania during 1915 has recently been given by Prof. Ricco, in continuation of the admirable series commenced by him in 1880 (Mem. Soc. Spett. Italiani, July-August, I9I7). The number of days of observation was I68, and the total number of prominences exceeding $15^{\prime \prime}$ in height was 1264 , giving a mean daily frequency of $7 \cdot 5$. The activity in the northern hemisphere was the greater, the respective numbers being 677 and 587 . The prominences were distributed almost symmetrically in the two hemispheres, few or none appearing in the polar and equatorial regions, while there were welldefined maxima in the zones $\pm 30^{\circ}-35^{\circ}$ and $\pm 50^{\circ}-55^{\circ}$. height, and one observed on May 5 reached $286^{\prime \prime}$. The mean latitude of all the prominences was $3^{8.65^{\circ}}$, which was rather lower than for the preceding year. There was a marked increase of activity as compared with I9I4.

\section{A COMMERCIAL IRON OF UNUSUAL PURITY.} F OR some time past the Shelton Iron, Steel, and facturing a commercial iron of unusual purity in the basic open-hearth furnace. This iron is guaranteed to be $99 \cdot 84$ per cent. pure, and has been placed on the market under the trade name "Armco Iron." It differs from wrought-iron in that it has been melted and cast, and thus contains much less slag, and from mild steel in that its carbon content is so low that no pearlite is present. It has been found, however, to possess a peculiar property which militates against its practical usefulness, viz. the property of a characteristic red-shortness, or brittleness, when subjected to mechanical treatment between certain limits of temperature. The reason for this peculiar behaviour, which is not shared by other forms of commercial iron and mild steel of high quality, has been investigated by Messrs. Brooke and Hunting, and their preliminary results were communicated in an interesting note to the recent meeting of the Iron and Steel Institute.

NO. 2503, VOL. IOO]
Very early in the history of the process it was found that this brittleness always appeared between certain fixed limits of temperature, which they place at from $900^{\circ}-800^{\circ} \mathrm{C}$; ; that on heating the iron to well above $\mathrm{Ac}_{3}$, and allowing it to cool, brittleness appeared, first at about $90^{\circ} \mathrm{C}$., and disappeared sharply at about $800^{\circ} \mathrm{C}$; ; and that above and below these temperatures the metal possessed an unusually high degree of ductility and malleability. In fact, they comment on its similarity when cold to copper in respect of malleability, thus emphasising also its resemblance to the electrolytic iron investigated in I9I3 by Stead and Carpenter.

The authors then proceeded to carry out systematic experiments on specimens of the iron quenched from various temperatures. They determined the tensile properties, and examined its structure and the type of fracture. Most interesting and illuminating results were obtained with the photographic records. Quenched from temperatures above $1000^{\circ} \mathrm{C}$., the structure was that of $\gamma$ iron with "martensitic" markings. As the quenching temperature fell this appearance altered, and the " $\gamma$ iron effect changed to a more definite ferrite form." The authors say that at about the $\mathrm{Ar}_{3}$ point a complete change occurred, the ferrite grains increased considerably in size, and at the junctions of many of the crystal boundaries a peculiar structure was observed which was "perfectly constant and always possessed the same characteristic ...
viz. a central structure more or less pearlitic and very clearly defined, surrounded by a space composed of ferrite, and the whole again surrounded by a definite boundary which connects up with adjacent crystal grains." This structure is clearly seen in the photographs published by the authors. They say that a very large number of experiments have been made, and that in every case this peculiar structure has appeared in exactly the same manner. On lowering the quenching temperature somewhat the structure gradually becomes less pronounced, and at just above $800^{\circ} \mathrm{C}$. it ceases to exist. Below $800^{\circ} \mathrm{C}$. it was never observed, and the structure was that of normal ferrite. The temperatures at which this material is precipitated and reabsorbed coincide so remarkably with the beginning and end of the zone of brittleness that a strong presumption has been established that herein lies the origin of the characteristic red-shortness of the iron. The authors suggest that it is a eutectoid, probably composed of iron carbide, phosphide, and sulphide, with possibly traces of manganese sulphide and ferrous oxide, and that it is thrown out of solution in a semi-liquid or plastic condition, causing the grains to be very loosely held together, and thus making the structure relatively weak. If the iron is quenched in the $900^{\circ}-800^{\circ} \mathrm{C}$. zone, no brittleness is observed in the static tests.

The authors have found that a sample of Swedish iron similar in purity to the above material also shows a brittle zone in the same temperature range, and that an iron containing carbon 0.06 per cent. and manganese o. Io per cent. shows no brittleness when manufactured in the same way as "Armco" iron. At one stage of the investigation they were inclined to connect the appearance of the material with the existence of dissolved oxide, since in one set of experiments they found it difficult to reproduce the characteristic structure, and this coincided with a remarkable absence of spots of "oxide material." Samples of metal, however, taken from the bath, just before tapping, when it was known to be in a super-oxidised condition, gave only a normal amount of eutectoid structure. Again, when complete deoxidation was attempted it still appeared. There is no reason, therefore, for connecting it with the presence of an unusuai amount of oxide, and the nature of the "eutectoid" 